\title{
KAGE: Fast alignment-free graph-based genotyping of SNPs and short indels
}

\author{
Ivar Grytten ${ }^{1,2 *}$, Knut Dagestad Rand ${ }^{1,2}$ and Geir Kjetil Sandve ${ }^{1,2}$
}

\author{
${ }^{*}$ Correspondence: \\ ivargry@ifi.uio.no \\ ${ }^{1}$ Department of Informatics, \\ University of Oslo, Gaustadalleen \\ 23 B, 0371 Oslo, Norway \\ Full list of author information is \\ available at the end of the article
}

\begin{abstract}
Genotyping is a core application of high-throughput sequencing. We present KAGE, a genotyper for SNPs and short indels that is inspired by recent developments within graph-based genome representations and alignment-free methods. KAGE uses a pan-genome representation of the population to efficiently and accurately predict genotypes. Two novel ideas improve both the speed and accuracy: a Bayesian model incorporates genotypes from thousands of individuals to improve prediction accuracy, and a computationally efficient method leverages correlation between variants. We show that the accuracy of KAGE is at par with the best existing alignment-free genotypers, while being an order of magnitude faster.
\end{abstract}

Keywords: genotyping; variant calling; pangenomes; graph genomes; alignment-free

\section{Background}

Recent studies such as the 1000 Genomes Project [1] have produced large catalogues of known human genetic variation. Researchers now have access to genotype information for thousands of individuals at known variant sites, providing unprecedented knowledge about how often genetic variants occur together at sub-population and individual levels.

Discovery and characterization of an individual's genetic variation have traditionally been done in two steps: 1) variant calling: discovering genetic variation in the genome, and 2) genotyping those called variants: determining whether each genetic variant is present in one or both of the chromosomes of the individual. Variant calling is a computationally expensive task, usually performed by first sequencing the genome of interest at high coverage, followed by mapping the sequenced reads to a reference genome and inferring variants according to where the mapped reads differ from the reference. It is now possible to skip the variant calling step, instead relying on catalogues of known genetic variation to directly genotype a given individual. For humans, the huge amount of already detected genomic variation means that a large amount $(>90 \%)$ of an individual's genetic variation can be detected this way [1]. Genotyping individuals at sites of known variation has in principle been performed already since the early 2000's based on SNP chips (microarrays). This has however been restricted to a limited set of fixed sites that a given chip has been designed to capture. While these chip-based techniques are still popular today due to their low cost, e.g. in GWAS studies [2], they are limited by chip architecture and thus only able to genotype about 1-2 million variants. Contrary to this, sequence-based genotyping techniques are, while being more expensive to perform, in theory able to genotype any genetic variant that has already been detected in the population. 
Sequence-based genotyping is traditionally performed by aligning sequenced reads to a reference genome and examining how the reads support genotypes locally at each variant $[3,4]$. While these methods generally have high accuracy, they are slow, mostly because read-mapping/alignment is computationally expensive. Also, since these methods require the mapping of reads to a reference genome, they have a tendency to perform poorly in regions where the reference genome is very different from the sequenced genome, such as in variant-rich regions. Furthermore, since reads are more likely to be correctly mapped when they are similar to the reference, reads supporting variant-alleles may be underrepresented among the mapped reads, resulting in a bias towards the reference alleles, a problem referred to as reference bias [5].

In an attempt to both speed up genotyping and avoid reference bias, several alignment-free approaches have emerged during the last few years $[6,7,8,9]$. These methods work by representing genetic variants by their surrounding kmers (sequences with length $\mathrm{k}$ covering each variant) and looking for support for these kmers in the sequenced reads. Since these methods do not map reads to a reference genome, they mitigate the problem of reference bias, and are usually computationally very efficient since kmer-lookup is fast compared to read mapping. However, these methods struggle when variant alleles cannot be represented by unique kmers, e.g. because a variant allele shares one or more kmers with another location in the genome. The genotyping method Malva[8] attempts to solve this problem by using larger kmers in cases where kmers are non-unique, but is still not able to genotype all variants using this approach. The more recent method PanGenie [10] takes the approach of Malva one step further and uses known population haplotype information to infer the likelihood of genotypes for variants that do not have unique kmers, such as variants in repetitive regions of the genome. PanGenie does this by using a Hidden Markov Model (HMM) with one state for each possible pair of haplotypes from the set of known haplotypes in the population. While this approach works well for relatively few input haplotypes and variants (PanGenie was tested using haplotypes from around 10 individuals and about 7 million variants), the number of states in the HMM increases quadratically with the number of haplotypes, and the method scales poorly when more than a few haplotypes are used. This means that the more than 5000 haplotypes and 80 million variants available in the 1000 Genomes Project cannot easily be utilised by an approach like PanGenie. Furthermore, PanGenie does not use kmer-information at all for variants with non-unique kmers.

We here describe KAGE - a new genotyper for SNPs and short indels that builds on recent ideas of alignment-free genotyping from Malva and PanGenie for computationally efficiency. KAGE implements two novel ideas for utilising all previously known haplotype information from repositories such as the 1000 Genomes Project in order to improve genotyping accuracy. We show that combining these ideas leads to a genotyper that is both more accurate and computationally efficient than existing alignment-free genotypers. 
Step 1: Index graph/variants with kmers

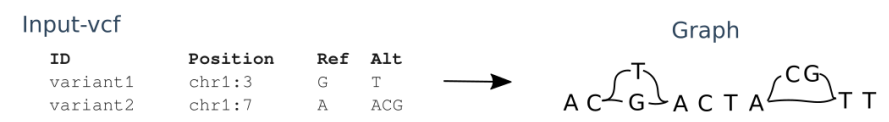

Kmer-index over variants in the graph $(\mathrm{k}=3)$

variant ID Ref-kmers Alt-kmers

$\begin{array}{lll}\text { variant1 } & \text { CTA } & \text { CGA } \\ \text { variant2 } & \text { ACG } & \text { ATT }\end{array}$

Step 2: Count kmers in reads

Step 3: Compute genotype-probabilities

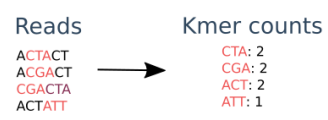

Using Bayes rule:

$\mathrm{P}($ gendypeelkner counts) $=\mathrm{P}($ kner counts /genotype) $* \mathrm{P}$ (gendype in population) / .

Figure 1 Overview of a typical alignment-free genotyping approach. First, kmers covering each allele of each variant is stored (step 1), e.g. by choosing kmers from a graph representation of the variants. All kmers from the reads are then collected (step 2), and genotype probabilities are computed using Bayes rule with genotype probabilities from a known population used as priors (step 3). The most likely genotype is chosen.

\section{Results}

We here present KAGE, which is based on two new ideas that are inspired by recent progress in alignment-free genotyping. We first show how each idea can improve genotyping accuracy for alignment-free genotyping methods, and then show that when these ideas are combined into KAGE, we get a graph-based alignment-free genotyper that is highly accurate and considerably more computationally efficient than existing alignment-free genotyping methods.

\section{Idea 1: Modelling expected kmer-counts from the population}

Alignment-free genotypers, like Malva and PanGenie, compute genotype likelihoods by counting the number of kmers from the read set that support each allele of every variant to be genotyped, as illustrated in Figure 1. A problem with this approach is that some variants may have alleles that are covered by kmers that also match elsewhere in the genome (for some or many individuals), making such variants difficult to genotype, as illustrated in Figure 2. We hypothesise that this problem may be addressed by modelling the expected match count for each individual kmer based on population data, such as data from the 1000 Genomes Project.

To test this hypothesis, we implemented a baseline genotyper that follows the scheme in Figure 1: each variant is represented by kmers, and the genotyper counts how many times each kmer exists in the read data sets, computing genotype probabilities using Bayes formula (naively assuming each kmer is unique in the genome). We also created a more sophisticated version of this genotyper, where we assume that each kmer exists with a given frequency in the population, and use this information when computing the probability of observing the given number of kmers 
bioRxiv preprint doi: https://doi.org/10.1101/2021.12.03.471074; this version posted December 20, 2021. The copyright holder for this preprint (which was not certified by peer review) is the author/funder, who has granted bioRxiv a license to display the preprint in perpetuity. It is made

for each variant (Methods). We benchmark both these methods against Malva. As shown in Figure 3, our naive method performs similarly to Malva, whereas the approach where expected kmer counts are modelled from the population clearly performs better. Although this is an experiment performed on a single small benchmark dataset, these findings provide an indication that the idea of comparing observed kmer counts to expected kmer counts based on a population may work.

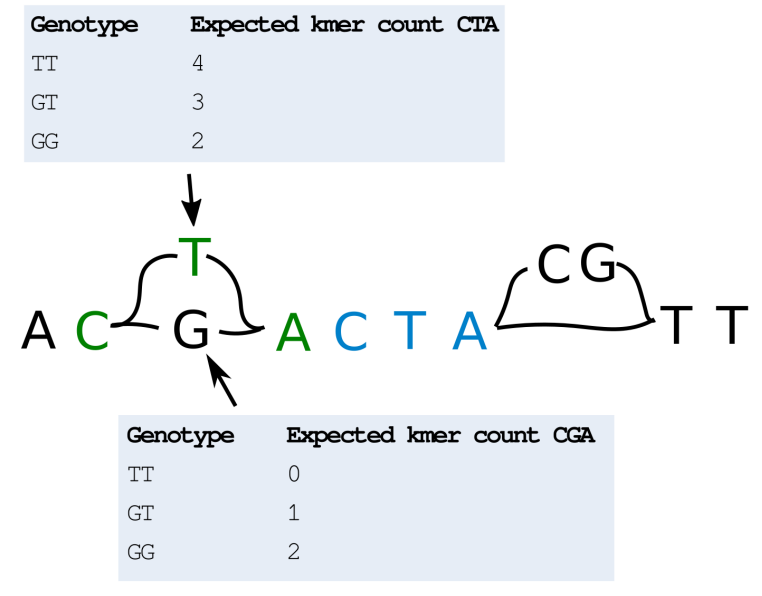

Figure 2 Graph with duplicate kmers. The SNP G/T has a kmer CTA (green) on the variant allele that also exists on the reference path (blue). If we observe each of the kmers CTA and CGA once in the read set, we might be fooled to believe that GT is the most likely genotype for this variant. However, when knowing that the kmer CTA is expected to occur at least once in the read data set, due to the duplication, we might conclude otherwise. This information can be used to adjust the probabilities used to compute the binomial probabilities of observing kmer counts given genotypes.

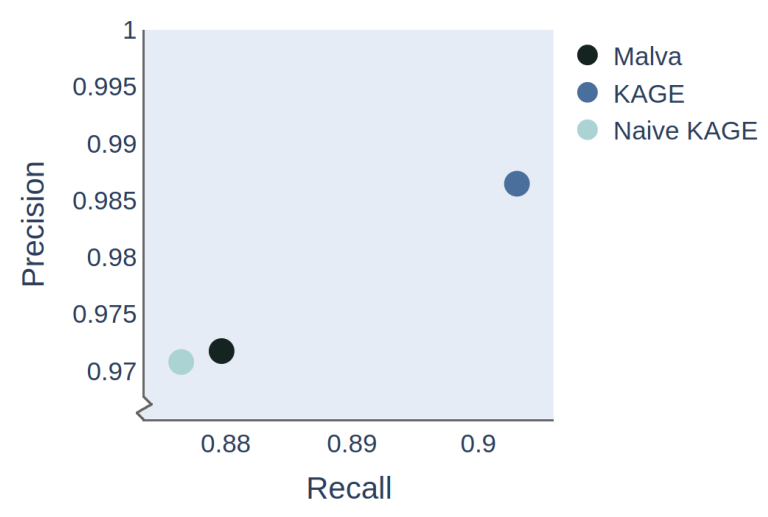

Figure 3 Genotype accuracy increases when expected kmer counts are modelled from the population. Our prototype alignment-free genotyper (Naive kage) performs a bit worse than Malva, as expected. However, when we compute expected kmer counts from the population, using 1000 Genomes data, and use these counts to compute the probability of observing the given kmers in our data, the genotyping accuracy greatly improves. 
Idea 2: Using a single variant to adjust the prior

While Malva uses the allele frequency from the population as priors for genotype likelihoods, PanGenie uses a more sophisticated approach where genotypes are inferred using a Hidden Markov Model (HMM), meaning that the predicted genotype of one variant provides information that can support the determination of the genotypes of other variants. While this approach may allow PanGenie to predict genotypes with higher accuracy than Malva, this method does not scale to handle a large number of known haplotypes, since the HMM must represent every possible pair of haplotypes as a separate state, making the number of HMM states grow quadratically with the number of haplotypes. We hypothesise that a simpler approach may be better for two reasons: First, a simple approach may be able to utilise all the haplotype information available (e.g. the 2548 individuals available from the 1000 Genomes project, rather than only a handful that the PanGenie approach would typically employ), and second, such an approach may be computationally faster than an HMM-approach that requires inference on hidden states. To test this hypothesis, we implement a way of efficiently computing prior probabilities for each genotype of each variant given genotype probabilities of other variants (Methods). We let every variant only depend on a single other variant, which leads to a very efficient way of genotyping all variants, and allows us to use as many underlying haplotypes as we would like. The determination of a single best suited helper variant for each known variant in the genome is performed only once, in a general pre-processing step. The genotyping of individuals then only needs to perform a fast lookup in this precomputed table. We test this approach against PanGenie, where PanGenie is run with a varying number of known haplotypes as input. In order to test PanGenie with as many haplotypes as possible from the 1000 Genomes Projects, we perform this experiment on a small test data set ( 5 million base pairs of chromosome 1).

The result of this experiment is shown in Figure 4, where it can be seen that the accuracy of both this approach (KAGE) and PanGenie increase with the number of known haplotypes. This experiment is performed on a small test dataset, so as to allow the inclusion of a larger number of individuals in Pangienie, and is not meant as a benchmarking of the methods per se. These findings provide an indication that the use of a single variant as prior still allows us to leverage information from a large number of individuals.

\section{A new graph-based alignment-free genotyper}

We combine the two ideas discussed above into a new alignment-free genotyper KAGE, and compare this genotyper in terms of running time, memory usage and accuracy against existing alignment-free genotypers as well as the most commonly used alignment-based genotypers. We follow the guidelines from "Best practises for benchmarking germline small-variant calls in human genomes" [11] and compare the output of each genotyper against a truth data set (similarly to how PanGenie and Malva assess their methods).

In the following experiments, we run PanGenie with 64 input haplotypes (from 32 individuals), since a larger number of haplotypes make PanGenie too slow and memory demanding when run on whole-genome data. We run KAGE with all of the 5096 
bioRxiv preprint doi: https://doi.org/10.1101/2021.12.03.471074; this version posted December 20, 2021. The copyright holder for this preprint (which was not certified by peer review) is the author/funder, who has granted bioRxiv a license to display the preprint in perpetuity. It is made

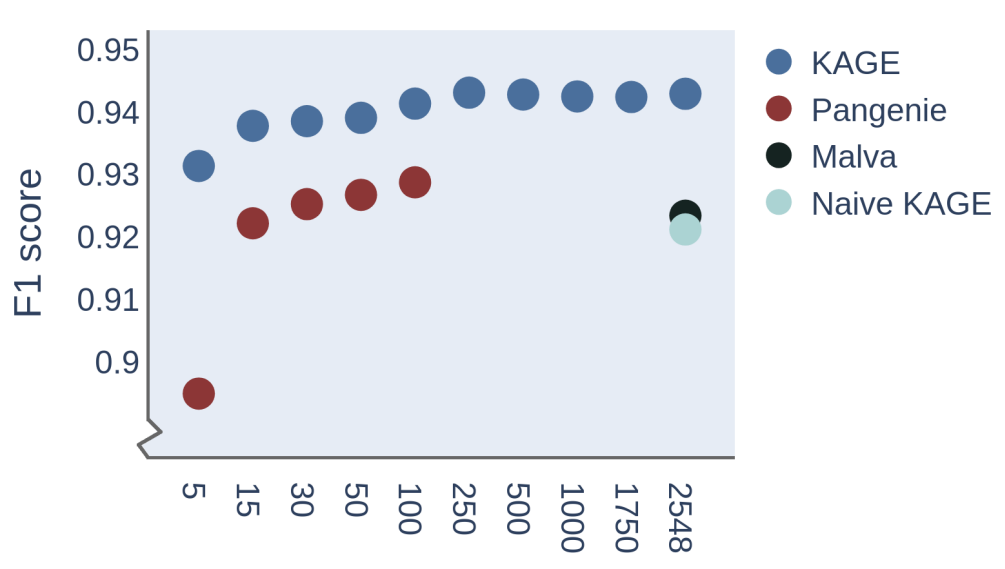

Number of individuals

Figure 4 Accuracy of Pangenie and KAGE as a function of the number of individuals included in the model on a small test dataset. Recall and precision (shown as F1 score) are computed for the predicted genotypes by the various methods on a small test dataset (genome size 5 million bp) with reads simulated from HG002, in order to test whether the accuracy of PanGenie and KAGE increases with a larger number of individuals in the model. The F1 score of Malva and "our naive approach" are shown as a single dot, since both of these only use the whole population genotype frequency when estimating genotype probabilities.

haplotypes available from the 1000 Genomes Project. When listing run times, we consider only the time used to genotype a given sample, not the time used to index the reference genomes, graphs, or variants, since such indices can be created once and then be re-used for genotyping an arbitrary number of samples. All experiments are based on genotyping variants from the 1000 Genomes Project using short reads as input. Accuracy is presented by recall and precision when predicted genotypes are compared against a truth dataset. Further details about these experiments can be found in the Methods section, and a Snakemake pipeline for reproducing all the results can be found at https://github.com/ivargr/genotyping-benchmarking.

In the experiments, we compare the genotypers listed in Table 1, which includes the alignment-based genotyper Graphtyper as well as all alignment-free genotypers we believe are relevant or commonly used. For comparison, we also run the commonly used variant caller GATK [3], which does not only genotype a specified set of variants, but tries to detect any variation from the reference. We include GATK so that the results of the genotypers can be contrasted to the alternative approach of performing full variant calling.

We genotype the individual HG002 from GIAB, which is an individual commonly used to benchmark variant callers and genotypers. The results can be seen in Table 2 and Figure 5. We ran the final version of KAGE also on another individual (HG006, also from GIAB), which was not consulted until after we finalised all method and parameter choices, and verified that the results are similar (Supplementary Table 2 and 3). The results from genotyping HG002 using 30x read coverage can be found in Supplementary Table 1, and are similar to the results from 15x coverage. The experiments performed show that KAGE is about as accurate as, or more accurate than, all the other genotypers and considerably faster than any of the methods. GATK comes out as clearly more accurate than the genotype methods, 
bioRxiv preprint doi: https://doi.org/10.1101/2021.12.03.471074; this version posted December 20, 2021. The copyright holder for this preprint (which was not certified by peer review) is the author/funder, who has granted bioRxiv a license to display the preprint in perpetuity. It is made

but is as expected considerably slower than most of the genotypers. KAGE is about as accurate as the alignment-based method Graphtyper while being more than 30 times faster.

\begin{tabular}{|l|l|l|}
\hline Genotyper & Alignment-free/alignment-based & Uses prior haplotype-information \\
\hline Malva & Alignment-free & No \\
\hline Bayestyper & Alignment-free & No \\
\hline PanGenie & Alignment-free & Yes \\
\hline Graphtyper & Alignment-based & No \\
\hline GATK & Alignment-based & No \\
\hline
\end{tabular}

Table 1 Overview of genotypers.

\begin{tabular}{lrrrrrrll}
\hline & Indels recall & Indels precision & Indels F1 & SNPs recall & SNPs precision & SNPs F1 & Runtime & Memory usage \\
\hline KAGE & 0.579 & 0.893 & 0.703 & 0.931 & 0.977 & 0.953 & 10 min & $14 \mathrm{~GB}$ \\
PanGenie & 0.562 & 0.928 & 0.7 & 0.907 & 0.981 & 0.943 & 3.6 hours & $113 \mathrm{~GB}$ \\
Bayestyper & 0.485 & 0.993 & 0.652 & 0.834 & 0.997 & 0.908 & 7.8 hours & $30 \mathrm{~GB}$ \\
Malva & 0.537 & 0.856 & 0.659 & 0.849 & 0.921 & 0.883 & 15.5 hours & $50 \mathrm{~GB}$ \\
Graphtyper & 0.568 & 0.941 & 0.708 & 0.909 & 0.993 & 0.949 & 5.2 hours & $23 \mathrm{~GB}$ \\
GATK & 0.898 & 0.96 & 0.928 & 0.966 & 0.981 & 0.973 & 9.3 hours & $60 \mathrm{~GB}$ \\
\hline
\end{tabular}

Table 2 Results from genotyping HG002.
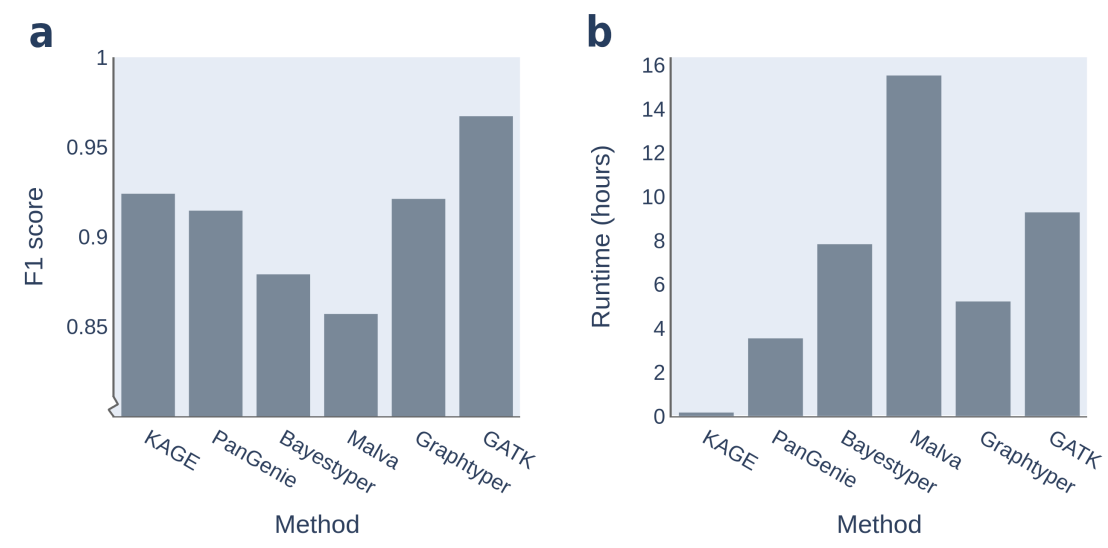

Figure 5 Accuracy and runtime of method Showing (a) the F1 score on SNPs and Indels combined and (b) the runtime of each of the methods when genotyping HG002 using read data with $15 \times$ coverage (16 compute cores).

\section{Discussion}

We find that KAGE has as high or higher accuracy than the other genotypers. PanGenie seems to perform similarly on indels, but performs slightly worse on SNPs. Malva performs worse than all the other methods, which we believe makes sense considering that Malva does not have a good way of dealing with variants represented by non-unique kmers. While PanGenie has decent accuracy, it requires more than 20 times the run time and considerably more memory than KAGE on the experiments we have run. KAGE is able to genotype a full sample with $15 \mathrm{x}$ coverage in only 10 minutes using 16 compute cores, while all the other methods require several hours. This means that with KAGE, it is now possible to genotype a sample quickly and easily on a standard laptop, such as in a clinical setting. The computational efficiency of KAGE also allows for a reduced energy footprint when 
large numbers of samples are to be genotyped. This is highly relevant given current plans of sequencing more than a million genomes in the coming years [12].

We confirm that performing full variant calling with a method such as GATK yields considerably higher accuracy than only genotyping a preset of specified variants. One should therefore be aware of this speed/accuracy tradeoff: genotyping a preset of known variants can be very efficient and give decent accuracy now that a lot of genomic variation is known. However, when the aim is to accurately discover as many variants in a sample as possible, and runtime is not an issue, a variant caller like GATK or DeepVariant [13] would be the best option. We note that GATK has higher accuracy in our experiments than what has been found in the previous benchmarks presented in the Graphtyper [4] and Malva [8] papers, where Malva and Graphtyper are both shown to have as good as and in some cases better genotyping accuracy than GATK. We believe this might be because GATK has been significantly improved in the last few years since these papers were published [3].

We are surprised to see that KAGE and PanGenie, which are completely alignment-free, are able to achieve very close accuracy to Graphtyper, which first maps and aligns all reads using BWA-MEM and then locally realigns all reads to a sequence graph. We believe the reason must be that KAGE and PanGenie exploit population information in a more sophisticated way when computing the genotype likelihoods. We speculate that PanGenie should be able to achieve even better accuracy by using more known haplotypes as input, but this would lead to very long run-time and high memory usage due to the quadratic complexity of the Hidden Markov Model used.

While Malva's strength is supposed to be a relatively low run-time, Malva is unfortunately not optimised to run on multiple compute cores/threads. We believe Malva would be able to run considerably faster if it was able to utilise multiple compute cores in the genotyping step. We observe that Bayestyper differs from the other methods by having a lower recall and a higher precision, meaning that Bayestyper is likely more conservative when genotyping. We were not able to tune Bayestyper to provide higher recall at the cost of lower precision.

There are a few limitations of KAGE. First, KAGE is for now only able to genotype SNPs and short indels, and it is important to note that our benchmarks only compare the tools on SNPs and short indels, while several of the methods have been developed also for structural variation. We believe, however, that the ideas that KAGE are based on can be generalised to structure variant calling with short reads, which would be an interesting opportunity for further work. Second, KAGE relies on a relatively good database of known variation from a population, meaning it would not work on species where such databases are not yet available. This does, however, also mean that we expect KAGE to potentially perform even better in the future when more genetic variation data from more individuals will be available. Finally, KAGE and the other kmer-based methods are not able to use information from paired-end reads. We do not consider this a major problem, however, since single-end sequencing is very common and cheaper to perform than paired-end sequencing. 


\section{Conclusions}

KAGE allows genotyping of SNPs and short indels by an alignment-free approach that is more than 20 times faster than existing methods, while offering competitive accuracy. We see KAGE as a highly timely contribution to the field, given the plans of large international consortia to sequence and genotype millions of genomes in the coming years.

\section{Methods}

\section{Model description}

The following describes the model and assumptions used in KAGE. We here assume all variants are biallelic variants, each with two alleles - a reference allele and an alternative allele. An individual can thus have the following three genotypes at a variant: Having the reference allele in both chromosomes (we refer to this as having the genotype $0 / 0$ ), having the alternative allele in both chromosomes $(1 / 1)$, or having the reference allele in one chromosome and the alternative allele in the other $(0 / 1)$. Note that we are not concerned with the phasing of genotypes, so $1 / 0$ and $0 / 1$ are considered as being the same genotype.

Assuming a variant of interest $i$, we define $P\left(G_{i}=g\right)$ as the probability of having the genotype $g$ at this variant, where $g$ is either $0 / 0,0 / 1$ or $1 / 1$. We let $K_{i}=$ $\left(K_{i r}, K_{i a}\right)$ be the observed kmer counts on the reference and variant allele of variant $i$. Every variant of interest $i$ has a preselected helper variant $h$ with kmer counts $K_{h}$. In order to find the most likely genotype for variant $i$, we want to calculate the posterior probabilities $P\left(G_{i} \mid K_{i}, K_{h}\right)$ :

$$
P\left(G_{i} \mid K_{i}, K_{h}\right)=P\left(K_{i} \mid G_{i}\right) \sum_{G_{h} \in G} P\left(G_{h}\right) P\left(G_{i} \mid G_{h}\right) P\left(K_{h} \mid G_{h}\right)
$$

In the above formula, $P\left(K_{i} \mid G_{i}\right)$ is the likelihood of observing the kmer counts $K_{i}$ on the variant of interest given a genotype $G_{i}$. The summation corresponds to a prior probability of the genotype $G_{i}$ given the observed counts on the helper variant and the population structure, with the individual probabilities corresponding to the following:

- $P\left(G_{h}\right)$ is the probability (over the population) of genotype $G_{h}$ at the helper variant.

- $P\left(G_{i} \mid G_{h}\right)$ is the probability (over the population) of having genotype $G_{i}$ on the variant of interest given genotype $G_{h}$ at the helper variant

- $P\left(K_{h} \mid G_{h}\right)$ is the likelihood of observing the kmer counts $K_{h}$ given genotype $G_{h}$ at the helper variant, similarly to $P\left(K_{i} \mid G_{i}\right)$.

The probabilities $P\left(G_{h}\right)$ and $P\left(G_{i} \mid G_{h}\right)$ are based on the observed genotypes on the specific variants $i$ and $h$ in the population, as well as priors (pseudo counts) that are based on all the variant-helper pairs across the genome. The full Bayesian model is detailed in the supplementary material.

The probability distributions for $P\left(K_{i} \mid G_{i}\right)$ and $P\left(K_{h} \mid G_{h}\right)$ are estimated by marginalizing over Poisson distributions for the unknown number of occurrences of the kmers in the genome (where occurrence uncertainty follows from variant uncertainty). If we knew the number of times the kmer of a variant occurs elsewhere 
in the genome $\left(D_{i} r\right.$, or $\left.D_{i} a\right)$, we could calculate the probability of observing the observed number of kmers in the sample as:

$$
P\left(K_{i r} \mid D_{i r}, G_{i}\right)=\operatorname{Pois}\left(K_{i r} ; \lambda\left(H_{r}\left(G_{i}\right)+D_{i r}+\epsilon\right)\right)
$$

where $\epsilon$ models noise (e.g. sequencing errors) and $H$ counts the number of copies of each allele for a genotype, i.e: $\left.H_{r}(0 / 0)\right)=H_{a}(1 / 1)=2, H_{r}(0 / 1)=H_{a}(0 / 1)=1$ and $H_{r}(1 / 1)=H_{a}(0 / 0)=0$. With uncertainty in the number of duplicates we get:

$$
P\left(K_{i} \mid G_{i}\right)=\sum_{D_{i}=0}^{\infty} P\left(K_{i} \mid D_{i}, G_{i}\right) P\left(D_{i}\right)
$$

The corresponding probabilities $P\left(K_{i a} \mid G_{i}\right), P\left(K_{h r} \mid G_{r}\right)$ and $P\left(K_{h a} \mid G_{a}\right)$ are calculated using the same setup. We refer to the Supplementary material for more details.

\section{Benchmarking genotypers}

We compared KAGE against the following other tools: GATK HaplotypeCaller version 4.2.3.0, Graphtyper version 2.7.1 (using the genotype subcommand), Malva version 1.3.1, PanGenie (commit ID da87f55cdccf31cd2c0008fd4848e33ba42021fc) and Bayestyper version 1.5. We also tried including Platypus in the comparison, but were not able to get the tool to work. Both Bayestyper and Malva use kmercounts from kmc[14]. We ran kmc version 3.1.1 and included singleton kmers (using the flag -ci1) for Bayestyper and Malva, as this seemed to give the best results. For Bayestyper and Malva, we used the suggested k specified by the tool documentation, which was 51 for Bayestyper and 31 (short kmers) and 45 (long kmers) for Malva. We ran KAGE and PanGenie with the recommended $\mathrm{k}=31$. While GATK has the option to genotype a specified set of variants, we did not use this option. The reason is that even when this option is specified, GATK will also call other alleles at the specified sites. We also experienced slower run time using this option. Thus, we chose to let GATK just do full variant calling, which is also what it is built for.

GATK and Graphtyper were run on reads mapped to GRCh38 using BWA-MEM [15] version 0.7.17-r1188. A Snakemake [16] pipeline for running all the experiments and a conda environment with all the necessary tools can be found at https://github.com/ivargr/genotyping-benchmarking. Reads were simulated using Graph Read Simulator (version 0.0.2) with substitution, deletion and insertion rate all set to 0.001 . When using experimental data all reads were obtained from the GIAB repository and downsampled to the given coverage used in the experiment. Exact data URLs can be found in the config file of the Snakemake pipeline.

Variants genotyped in the experiments are from the 1000 Genomes Project v2a. Only variants with allele frequency $>0.1 \%$ were used. We used Hap.py version 0.312 [11] to compute accuracy (precision and recall) against a truth dataset. 
bioRxiv preprint doi: https://doi.org/10.1101/2021.12.03.471074; this version posted December 20, 2021. The copyright holder for this preprint (which was not certified by peer review) is the author/funder, who has granted bioRxiv a license to display the preprint in perpetuity. It is made

\section{Software implementation}

KAGE was implemented using Python 3, and some run-time critical code (kmer lookup) were written in Cython. All code developed as part of this method has been modularized into four Python packages, each available through the PyPi package repository: kage-genotyper (main tool for genotyping), graph_kmer_index (building and using the kmer indexes necessary for the genotyping), kmer-mapper (counting kmers) and obgraph (building and working with the sequence graphs). KAGE with all dependencies can be installed directly by installing only the kage-genotyper package.

Ethics approval and consent to participate

Not applicable

\section{Consent for publication}

Not applicable

\section{Competing interests}

The authors declare that they have no competing interests.

\section{Funding}

This work was supported by the Centre for Computational Inference in Evolutionary Life Science (CELS). We also acknowledge generous support by the Research Council of Norway for an IKTPLUSS project (\#311341) to KR and GKS.

\section{Author contributions}

IG, KDR and GKS drafted the manuscript. IG and KDR developed the software. IG, KDR and GKS contributed to the conception and design of the study, and to the interpretation of data. All authors have read and approved the final version of the manuscript.

\section{Availability of data and materials}

The datasets generated and/or analysed during the current study are available through the following git repository: https://github.com/ivargr/genotyping-benchmarking (version 0.0.1). An index that can be used with KAGE to reproduce the experiments is available at https://zenodo.org/record/5786313/files/index_2548all.npz. KAGE is available at https://github.com/ivargr/kage (version 0.0.18, GNU General Public Licence v3.0).

\section{Author details}

${ }^{1}$ Department of Informatics, University of Oslo, Gaustadalleen 23 B, 0371 Oslo, Norway. ${ }^{2}$ Centre for Bioinformatics, University of Oslo, Gaustadalleen 30, 0373 Oslo, Norway.

\section{References}

1. 1000 Genomes Project Consortium and others: A global reference for human genetic variation. Nature 526(7571), 68 (2015)

2. Korte, A., Farlow, A.: The advantages and limitations of trait analysis with GWAS: a review. Plant methods 9(1), 1-9 (2013)

3. Poplin, R., Ruano-Rubio, V., DePristo, M.A., Fennell, T.J., Carneiro, M.O., Van der Auwera, G.A., Kling, D.E., Gauthier, L.D., Levy-Moonshine, A., Roazen, D., et al.: Scaling accurate genetic variant discovery to tens of thousands of samples. BioRxiv, 201178 (2017)

4. Eggertsson, H.P., Jonsson, H., Kristmundsdottir, S., Hjartarson, E., Kehr, B., Masson, G., Zink, F., Hjorleifsson, K.E., Jonasdottir, A., Jonasdottir, A., et al.: Graphtyper enables population-scale genotyping using pangenome graphs. Nature Genetics 49(11), 1654-1660 (2017)

5. Martiniano, R., Garrison, E., Jones, E.R., Manica, A., Durbin, R.: Removing reference bias and improving indel calling in ancient DNA data analysis by mapping to a sequence variation graph. Genome Biology 21(1), 1-18 (2020)

6. Shajii, A., Yorukoglu, D., William Yu, Y., Berger, B.: Fast genotyping of known SNPs through approximate k-mer matching. Bioinformatics 32(17), 538-544 (2016)

7. Sun, C., Medvedev, P.: Toward fast and accurate SNP genotyping from whole genome sequencing data for bedside diagnostics. Bioinformatics 35(3), 415-420 (2019)

8. Denti, L., Previtali, M., Bernardini, G., Schönhuth, A., Bonizzoni, P.: MALVA: genotyping by Mapping-free ALlele detection of known VAriants. Iscience 18, 20-27 (2019)

9. Sibbesen, J.A., Maretty, L., Krogh, A.: Accurate genotyping across variant classes and lengths using variant graphs. Nature Genetics 50(7), 1054-1059 (2018)

10. Ebler, J., Clarke, W.E., Rausch, T., Audano, P.A., Houwaart, T., Korbel, J., Eichler, E.E., Zody, M.C., Dilthey, A.T., Marschall, T.: Pangenome-based genome inference. bioRxiv (2020)

11. Krusche, P., Trigg, L., Boutros, P.C., Mason, C.E., Francisco, M., Moore, B.L., Gonzalez-Porta, M., Eberle, M.A., Tezak, Z., Lababidi, S., et al.: Best practices for benchmarking germline small-variant calls in human genomes. Nature Biotechnology 37(5), 555-560 (2019) 
bioRxiv preprint doi: https://doi.org/10.1101/2021.12.03.471074; this version posted December 20, 2021. The copyright holder for this preprint (which was not certified by peer review) is the author/funder, who has granted bioRxiv a license to display the preprint in perpetuity. It is made

12. Saunders, G., Baudis, M., Becker, R., Beltran, S., Béroud, C., Birney, E., Brooksbank, C., Brunak, S., Van den Bulcke, M., Drysdale, R., et al.: Leveraging European infrastructures to access 1 million human genomes by 2022. Nature Reviews Genetics 20(11), 693-701 (2019)

13. Poplin, R., Chang, P.-C., Alexander, D., Schwartz, S., Colthurst, T., Ku, A., Newburger, D., Dijamco, J., Nguyen, N., Afshar, P.T., et al.: A universal SNP and small-indel variant caller using deep neural networks. Nature Biotechnology 36(10), 983-987 (2018)

14. Kokot, M., Długosz, M., Deorowicz, S.: KMC 3: counting and manipulating k-mer statistics. Bioinformatics 33(17), 2759-2761 (2017)

15. Li, H.: Aligning sequence reads, clone sequences and assembly contigs with BWA-MEM. arXiv preprint arXiv:1303.3997 (2013)

16. Mölder, F., Jablonski, K.P., Letcher, B., Hall, M.B., Tomkins-Tinch, C.H., Sochat, V., Forster, J., Lee, S., Twardziok, S.O., Kanitz, A., et al.: Sustainable data analysis with Snakemake. F1000Research 10 (2021)

\section{Additional Files}

Additional file 1

Includes results for additional experiments and further details of method. 